\title{
Currículo sobre la enseñanza del pasado reciente y violencia política en Colombia. ${ }^{1}$
}

por Diego H. Arias Gómez

Universidad Distrital Francisco José de Caldas, Colombia

dhariasg@udistrital.edu.co

y Martha C. Herrera

Universidad Pedagógica Nacional, Colombia

malaquita10@gmail.com

Recibido: 31/07/2018 - Aceptado: 27/08/2018

\section{Resumen}

El presente escrito recoge los hallazgos de dos investigaciones centradas en el tratamiento de la violencia política y el conflicto armado en el campo educativo y escolar, específicamente en las particularidades del abordaje de la historia reciente en la escuela colombiana. A partir de la revisión de documentos académicos y la percepción de docentes en ejercicio se pudieron identificar las dificultades y potencialidades que el tema concita, especialmente aquellas relacionadas con los retos de posicionar la categoría historia reciente en el país, la importancia de las apuestas ético-políticas de los docentes para las definiciones curriculares y la ambigüedad de la normativa educativa respecto al tema del conflicto armado interno.

Palabras claves

enseñanza de la Historia, enseñanza de las ciencias sociales, formación política, estudios sociales, violencia política

\section{Curriculum on the teaching of the recent past and political violence in Colombia.}

\section{Abstract}

The present document collects the findings of two investigations focused on the treatment of political violence and armed conflict in the educational and school field, specifically in the particularities of the approach to recent history in the Colombian school. From the review of academic documents and the perception of teachers in practice, it was possible to identify the difficulties and potentialities that the subject arouses, especially those related to the challenges of positioning the recent history category in the country, the importance of ethical bets Teachers' policies for curricular definitions and the ambiguity of educational regulations regarding the issue of internal armed conflict.

\section{Keywords}

History teaching, social science teaching, political training, social studies, political violence 


\section{Introducción}

La enseñanza de la historia reciente se ha convertido en una de las problemáticas más acuciantes en las políticas educativas en el continente latinoamericano y ha suscitado enormes desafíos en el campo del currículo, en especial a partir de las décadas del 80 y del 90 del pasado siglo, debido a una serie de reformas constitucionales que coincidieron con la implementación de leyes generales de educación en muchos países de la región, en las que se plasmaron diversas demandas en torno a las políticas públicas referentes a la universalización de la educación y a los derechos humanos, varias de las cuales estuvieron en conexión con políticas de la memoria que buscaron encarar pasados marcados por regímenes dictatoriales, como Chile y Argentina, o por picos represivos en países de democracia restringida, como Colombia.

Las reformas curriculares expresadas en el campo de las políticas educativas conllevan retos que permean diversos escenarios e involucran distintos actores sociales y educativos, señalando las dificultades inherentes a la puesta en marcha de las políticas públicas y su ejecución en los establecimientos educativos, así como su apropiación por parte de directivas escolares, docentes, estudiantes y padres de familia. Lo anterior, encuentra mayores desafíos cuando se trata de componentes curriculares que atañen a la historia reciente, debido a que muchas de las problemáticas a ser tratadas en este ámbito forman parte de un pasado que involucra de manera directa al arco generacional que interactúa en el tiempo presente, pues muchos de los agentes educativos han vivido algunos de los acontecimientos relacionados con esos sucesos, sin que se haya tomado todavía la distancia necesaria para su procesamiento, al tiempo que en el campo académico apenas se están gestando formas de comprensión e inteligibilidad que ayuden a su tramitación histórica y social.

De ese modo, en el presente artículo nos interrogamos, con base en dos investigaciones, una llevada a cabo en la Universidad Distrital Francisco José de Caldas y otra en la Universidad Pedagógica Nacional, en torno a algunas de las aristas de esta problemática y los diversos tratamientos que le han sido dados en los escenarios educativos. Para ello el escrito aborda algunos aspectos de la política educativa sobre la enseñanza de la historia reciente, así como las apropiaciones curriculares que hacen algunos docentes sobre esta problemática, tales como el manejo del tiempo y el espacio, los tópicos seleccionados y las fuentes privilegiadas para su trabajo. Se cierra con unas conclusiones que condensan algunas discusiones expuestas a lo largo del escrito. Vale aclarar que estos análisis son exploratorios y por tanto no son proclives de ser generalizados para la escuela colombiana, cuyo propósito y metodología desborda los alcances del presente escrito.

\section{Currículo y enseñanza de la historia reciente}

El currículo debe ser comprendido de manera amplia como un espacio social en movimiento que condensa múltiples propósitos de carácter social, cultural y educativo que obedecen a contextos y coyunturas históricas específicas, los cuales encuentran formas de realización en diversos componentes que atañen al ámbito escolar, dentro de los cuales se podría aludir a los contenidos prescritos, a los libros de textos, las guías didácticas, las programaciones de cada institución, las tareas de aprendizaje, las evaluaciones y la cotidianidad escolar, entre otros.

Para Goodson (1991), más que entender el currículo como lo escrito en los documentos o como lo practicado en el aula, sería pertinente volcarse en el estudio de su construcción social para leer los diversos aspectos que acabamos de mencionar. De allí que este autor prefiera comprender el currículo como resultado de la conflictiva invención de una tradición, cuyos avatares suceden fundamentalmente fuera de la escuela. En sus palabras,

... a no ser que analicemos la construcción del currículum tendremos la tentación de aceptarlo tal como es y de buscar variables dentro del aula o, por lo menos, dentro del ámbito escolar individual. Estaríamos aceptando como 'tradicionales' y 'predeterminadas' versiones de currícula que, 
analizadas más detenidamente, pueden considerarse como la culminación de un conflicto largo y continuo. (Goodson, 1991:13)

Este tipo de acercamiento es profundizado por Gimeno, quien afirma que la idea de currículo materializa una transmisión cultural deliberada que se hace de una generación a otra. Así, "educación escolar e instituciones creadas para llevarla a cabo son respuestas prácticas a necesidades de un tipo específico de sociedad, a determinados modelos de vida y a una cierta jerarquía de valores" (Gimeno (1998:179), lo que quiere decir no solo que los contenidos que circulan oficialmente en la escuela son producto de ciertas apuestas institucionales y legales, sino que el currículo tiene que ver con todo aquello que sucede en la escuela. En esta dirección, Gimeno y Pérez (1993) indican que el currículo debe responder a algunas preguntas, tales como ¿qué objetivos se persiguen con la enseñanza?, ¿qué valores, actitudes y conocimientos están implicados en la enseñanza?, ¿quién define lo que se debe enseñar?, ¿por qué enseñar unas cosas y desechar otras?, ¿a qué intereses sirven estos conocimientos?, ¿con qué recursos metodológicos y materiales enseñar?, entre otras.

Esta conflictiva construcción del currículo se pone en evidencia, particularmente, en el tratamiento escolar de los pasados recientes vinculados a la violencia política, no solo por la falta de consensos sobre los temas a abordar en el aula, sino porque su tratamiento despierta diferentes tipos de conflicto en la sociedad y, por supuesto, en la escuela. En los últimos lustros se ha instalado en la literatura educativa y de las ciencias sociales el concepto de enseñanza de la historia reciente o del pasado reciente para referirse a los fenómenos de no lejana sucesión que han afectado la dinámica de las sociedades, especialmente latinoamericanas, y que han sido incorporados en el currículo. En otros contextos se habla de enseñanza de cuestiones socialmente vivas (Pagés y Santisteban, 2013) o de enseñanza de aspectos históricamente relevantes (Falaize, 2010).

Independientemente del nombre que se otorgue, un ingrediente fundamental en la mirada sobre estos acontecimientos está cruzado por las opciones ético-políticas que los gestores de las políticas educativas y docentes hacen sobre la selección de los contenidos y las metodologías sobre las temáticas de cierto pasado a enseñar (Carretero, 2005). Por ello es importante analizar la serie de motivaciones desplegadas en los procesos de transmisión generacional, pues a partir de ello es posible "conocer qué sentidos del pasado trabaja de manera intencional una sociedad para dejar su legado y, también, qué parte de esa herencia será olvidada, silenciada o permanecerá como un todavía no en espera de ser reactivada" (Mendoza, 2015:59).

Para la realidad latinoamericana, los estudios sobre la historia reciente y la memoria, emergieron como categorías analíticas en medio de los procesos de transición democrática en aquellas naciones que padecieron regímenes dictatoriales, autoritarios o represivos, en tanto estrategia social de recomposición y reconstrucción del tejido social fracturado, a la vez que bandera por la defensa de los derechos humanos, el derecho a la verdad sobre los crímenes cometidos y como eje en el que convergieron organizaciones de la sociedad civil (Dussel, 2002; Jelin, 2003). Especialmente por la experiencia de las dictaduras en el Cono Sur, se vitalizó la necesidad de plantear una lucha por la construcción de los significados de hechos del pasado desde la escuela (Jelin y Lorenz, 2004; Funes, 2006; Higuera, 2012).

Allí, la enseñanza de la historia y de las ciencias sociales asumió el estudio del pasado reciente como una lucha política por cuestionar verdades oficiales y hacer visibles versiones subalternas por algún tiempo desconocidas. Además, el estudio de la historia reciente buscó propiciar en las nuevas generaciones posicionamientos morales y ético-políticos frente a conflictos pretéritos y presentes de grupos y fuerzas alternativas que chocaron contra el poder hegemónico, es decir, la enseñanza de la historia reciente y de la violencia política padecida en muchas naciones se utilizó en algunos contextos como campo estratégico de formación política escolar (Arias y Ruiz, 2013).

Clín \& Asaciados. La historia enseñada. Julin-Diciembre ZOI8 (27) ISSN 2362-3063 (digital), pp. I8-29. UNL - UNLP 
Aunque tal incorporación al currículo de la historia reciente no ha estado exenta de problemas e incongruencias (De Amézola, 2008), su presencia es un hecho y su impacto se ha sentido en muchos países, golpeados por graves conflictos sociopolíticos de trascendencia nacional e internacional. La escuela y la enseñanza de lo histórico son interpeladas por impactantes fenómenos de reciente ocurrencia cuyo cariz contiene alta polémica social. Esta intromisión incómoda del pasado cercano en la escuela dramatiza con fuerza el pretendido carácter no neutral que con frecuencia se ha supuesto deben tener los contenidos de la enseñanza de lo histórico-social (Arias, 2005), y que ahora, a propósito de hechos dolorosos para la sociedad, tiene un importante papel para ayudar a garantizar que tales acontecimientos no se repitan. Por ello, se puede afirmar que no basta con repudiar ciertos hechos, no es suficiente repetir fórmulas de conjuro contra un pasado ominoso (Nunca más, ;Basta ya!), más bien, el reto es acompañar a los estudiantes en la construcción de una perspectiva que les permita comprender por qué sucedieron, cómo situarse de manera ética y política frente a ellos y cómo avizorar el presente y el futuro tomando distancia frente a ellos (Levín, 2007; Herrera y Vélez, 2014).

En esta línea, según Kriger (2011), la tradicional misión de la escuela que enfrenta lo cognitivo y lo identitario, se tensiona aún más con la enseñanza del pasado reciente y pone en evidencia la importancia del papel de la memoria en las actuales sociedades, especialmente las que han pasado o están tramitando memorias traumáticas. Así, los contenidos y orientaciones de este pasado incorporado en la escuela son debatibles y, sobre todo, objeto de disputa por diferentes agencias sociales. ¿Quién define lo que debe ser enseñado en la escuela respecto a las últimas décadas?, ¿con qué insumos cuentan los docentes para interpretar el pasado reciente que llevan al aula?, y aún más, ¿qué contenidos privilegian enseñar de los pasados traumáticos y por qué?, son algunas preguntas que vale la pena formularse respecto a esta realidad y sus posibilidades de enseñanza.

Los pasados traumáticos que se resisten a pasar, incomodan al presente, son problemáticos para la sociedad y para el tipo de escuela que cuestiona las versiones oficiales, consignadas en los libros de texto, en plataformas virtuales del Estado o en lineamientos curriculares, mayormente, cuando este pasado pone en entredicho la legitimidad misma de la institucionalidad (Carretero y Borrelli, 2010). La enseñanza de la historia reciente, con frecuencia, puede molestar al poder y a grupos ligados a él que han cohonestado con hechos que violan los derechos fundamentales de personas y comunidades y que, adicionalmente, han impuesto versiones acomodadas sobre lo sucedido con los influyentes medios que tienen a su disposición (Herrera y Pertuz, 2016).

En suma, la enseñanza de la historia reciente tensiona la lógica de las tradicionales disciplinas escolares ocupadas del abordaje de lo histórico y lo social, a la vez que problematiza el escenario escolar al incorporar unos contenidos complejos y polémicos ligados a sucesos de reciente ocurrencia, que por tanto desafían los currículos prescritos y cuestionan los órdenes culturales y políticos de la sociedad.

\section{Los tiempos y espacios escolares}

Con la instauración de los sistemas escolares y la institucionalización de la escuela bajo la tutela del Estado se fueron regulando el tiempo y el espacio de los infantes, lo que fue dando pie a la caracterización de un tiempo y un espacio social que pasó a tener vida propia en el ámbito de la escuela. Para Escolano, "tiempo y espacio son, además de mediaciones básicas en la construcción histórica de la infancia, dos categorías esenciales de la cultura de la escuela, esto es, de los códigos que han creado los enseñantes para reducir a determinadas reglas la actividad académica y la propia profesión docente" (Escolano, 2000:13). Según este autor,

... el orden del tiempo en educación, además de ser el mejor registro de la memoria de la escuela, es desde luego un pattern de la construcción social de la realidad, una estructura constitutiva de la infancia, una ratio que configura su currículum en el más amplio sentido, que puede incluir no sólo el programa académico, sino también su alternancia con los ocios y otros aspectos de la 
cotidianidad. Esta "escritura" del tiempo revelaría, pues, los trabajos y los días de la infancia y de los maestros, los escenarios en que éstos tuvieron lugar, los ritmos de la duración escolar y de la vida de toda la colectividad en cada época, la interacción entre las prescripciones educativas y la liturgia, las costumbres y las actividades económicas y culturales de la sociedad. (Escolano, 2000:15).

De este modo, el espacio y el tiempo que ha sido asignado a los temas relacionados con la historia reciente en la escuela han estado en tensión y competencia con otros tópicos que se han considerado usualmente de mayor importancia. Por lo general casi siempre se asigna en el currículo un tiempo y espacio mayor a períodos históricos anteriores, por tanto este se aborda de manera "casual", o casi nunca se logra llegar al momento presente, o se llega de manera rápida al final del período escolar. Lo anterior debido a una tradición secular que partía de la idea de que "todo tiempo pasado fue mejor", pero también a lo que mencionamos al comienzo de este artículo relacionado con las dificultades sociales por tramitar los pasados recientes y procesarlos de manera adecuada. Parece que aquello postulado y de corriente uso respecto a que la escuela debe ser neutral logra ser defendido cuando se habla de un pasado no tan cercano, porque supuestamente no hay apegos, aunque no logra verse que toda selección del pasado conlleva una elección ética y política, pero esta posición se torna problemática cuando se hace referencia a acontecimientos que han incidido de manera directa en las vidas de las generaciones que conviven en el tiempo presente y cuyas expectativas y horizontes de futuro confluyen de manera tumultuosa en los escenarios escolares.

Buena parte de los docentes cuyas prácticas han sido auscultadas por las diversas investigaciones coinciden en que el tiempo y los espacios escolares que pueden dedicar a la enseñanza de contenidos referidos a la historia reciente son muy cortos, fundamentalmente porque hay una cantidad exagerada de temas para abordar en el año lectivo, y porque la trascendencia de los asuntos controversiales no es mayor respecto a otros, en el sentido que los contenidos sobre violencia política y conflicto social y armado comparten el mismo o menor nivel de jerarquía que los otros tópicos del currículo. Por otra parte, la mayoría de docentes coincide en afirmar que noveno grado es el curso en el que suele enseñarse la historia reciente, por ser un contenido específico de la historia de Colombia, tema usualmente destinado para este grado.

Algunos docentes manifiestan la dificultad de articular los contenidos de historia y geografía, no solo por los problemas del tiempo escolar asignado, sino por la falta de unidad en las políticas educativas al respecto y por las rutinas establecidas a partir de las prácticas docentes. Este dilema pone de manifiesto una serie de inconsistencias en la práctica de las ciencias sociales escolares del país y en la normativa que la regula respecto a la integración curricular, lo cual contribuye a que los docentes del área tomen varias vías de solución. Aunque este no es el lugar para ahondar en ello (Arias, 2015), vale decir que tanto los Lineamientos (MEN, 2002), como los Estándares (MEN, 2004) y los anunciados Derechos Básicos de Aprendizaje del área (MEN, 2017), en su conjunto, apuntan a aspectos epistemológica y metodológicamente distintos, pues obedecen a coyunturas y a escuelas teóricas disímiles, sin mencionar la falta de articulación con las políticas de formación docente que, en términos generales, sigue siendo resuelta con una lógica eminentemente disciplinar, en este caso, con énfasis en historia y geografía; por tanto, la mayoría de docentes del país privilegian en su enseñanza alguna de estas dos disciplinas o ven la integración curricular como un dilema insalvable ${ }^{2}$.

El asunto del tiempo escolar dedicado al estudio en las escuelas no es algo menor, pues finalmente el proceso de escolarización se vive para docentes y estudiantes como una permanencia en el tiempo y en el espacio. El espacio escolar es un lugar signado por determinada arquitectura que tiene unos límites con el afuera, estos lugares están delimitados para ciertos propósitos: las aulas, el patio, las oficinas administrativas, los corredores, los baños. Para Meirieu, la organización de los espacios y los tiempos en la escuela deben ser plenamente identificables "porque el aprendizaje no puede ser una

Clín \& Asociados. La historia enseñada. Julio-Diciembre 2018 (27) ISSN 2362-3063 (digital), pp. I8-29. UNL - UNLP 
especie de río caótico que arrastraría a los alumnos, quienes pasarían de una etapa a otra sin comprender lo que les sucede" (Meirieu, 2004:217). Se va a la escuela para habitar un espacio y para vivir un tiempo en forma organizada.

La vida escolar, así, tiene sentido en tanto se ocupan y se habitan unos espacios en los que se espera que el aprendizaje suceda. Pero también está el tiempo, esos fragmentos que engloban la existencia en una sucesión permanente de aconteceres que se miden, se catalogan y se etiquetan, y que, para la escuela, significan el llenado del estar siendo en ella. Es más, se asiste a la escuela para vivir un tiempo determinado del día, del mes y de la vida. Así como se diseñan y se dividen los espacios para promover actitudes, comportamientos y pensamientos en los escolares, también se elaboran y se recortan los tiempos para generar determinados aprendizajes (Escolano, 2000).

Respecto al uso de espacios diferentes al aula para desplegar estrategias pedagógicas en torno a la enseñanza del pasado reciente, no se encuentra en las investigaciones muchos detalles, más allá del uso de escenarios como el teatro o los patios escolares en donde en ocasiones se llevan a cabo conferencias, conciertos u obras de teatro alusivas a este tipo de problemáticas, así como algunas salidas pedagógicas esporádicas a museos. En este sentido, en contraste con países como Argentina o Chile, en los cuales de manera progresiva los lugares de la memoria se han venido incorporando en las políticas educativas como parte de una pedagogía pública de la memoria, en el país apenas se comienza a hablar de esta necesaria articulación y, por lo tanto, de la importancia de incentivar a que las escuelas y los maestros incorporen este tipo de escenarios en la enseñanza (Herrera y Pertuz, 2016).

La enseñanza del pasado reciente es posible porque ocupa un tiempo en el calendario y en la rutina escolar. Ella disputa con otras asignaturas y deberes escolares el fugaz y veloz tiempo escolar que nunca es suficiente, que siempre es escaso. Por ello, en el marco de las afugias de los docentes por completar un plan de estudios enciclopédico, el tiempo nunca alcanza; empero, solo algunos, que miran más allá de las obligaciones institucionales o legales logran sobreponerse al frenesí temático que impone la tradición, los libros de texto o el currículo oficial, para acumular unas dosis adicionales de tiempo y espacio y dedicarse con sus estudiantes a comprender qué y por qué pasó lo que pasó en la historia de las últimas décadas. Estos son docentes, que logran sobrepasar la rutina y no se pierden en medio de la turbulencia escolar a la que son sometidos, son docentes arriesgados y conscientes de los costos que ello comporta, y que vuelven realidad las palabras de Meirieu: "la habilidad del marino y la experiencia del pedagogo les permiten, a veces, prescindir de uno o varios jalones, ir más rápido hacia el fin que hay que conseguir, incluso si ello implica, inevitablemente, riesgos más importantes" (Meirieu, 2004:212).

\section{Algunos tópicos sobre violencia política en la escuela}

El asunto de la historia reciente en la escuela se juega en la posibilidad o no de incorporar unos contenidos social y políticamente sensibles en el aula (Levín, 2007), para el caso del presente artículo, aquellos referidos a la violencia política y al conflicto interno de las últimas décadas; por ello, explorar las temáticas que escogen los profesores al momento de hacer la pregunta ¿qué temas de la violencia política aborda en su clase? es central para caracterizar la enseñanza del pasado reciente en el país y profundizar en las razones por las cuales se seleccionan unos contenidos y se desechan otros. En este punto, para la enseñanza del pasado reciente, importa analizar lo que se dice y lo que se acalla, lo que queda y lo que ha cambiado, lo que se privilegia y lo que se oblitera, respecto a un tema que concita un interés particular por apuntalar temas de alta sensibilidad social.

En términos generales los temas abordados por los maestros en torno a violencia política y el conflicto interno de las últimas décadas, se pueden desglosar grosso modo en los siguientes: Constitución de 1886, Guerra de los Mil Días, separación de Panamá, bipartidismo, masacre de las Bananeras, Bogotazo (1948), dictadura de Rojas Pinilla, Frente Nacional, nacimiento de grupos guerrilleros, paramilitarismo, narcotráfico, toma del Palacio de Justicia, procesos de paz, apertura 
económica, desplazamiento, guerras mundiales, Holocausto y dictaduras en América Latina (Arias, 2017; Herrera, Ortega, Cristancho y Olaya, 2013).

Al respecto, llama la atención la ausencia de un referente cronológico común y la diversidad de aspectos amparados bajo la categoría de historia reciente en la cual cabe una amplia constelación conceptual sin límites espaciales o temporales precisos. También es sintomático que solo pocos profesores manifiestan trabajar en el aula los procesos de paz con la guerrilla, siendo que la agenda pública del país de las últimas décadas ha estado copada inicialmente por los diálogos con los paramilitares, bajo la administración de Uribe Vélez, y luego por los diálogos con las FARC, con Santos.

La mayoría de docentes no tienen una referencia definida respecto al tema de la violencia política en Colombia, en el sentido de ubicarla en las últimas décadas y en torno a determinados acontecimientos ligados al conflicto social y armado, especialmente con el Bogotazo de 1948, como sí parece ocurrir con la productividad en el ámbito académico, que sitúa en aquel nefasto acontecimiento la génesis de una cadena de violencias que se prolonga hasta inicios del siglo $\mathrm{XXI}^{3}$.

Sin embargo, tal consenso es relativo ante la reciente proliferación de estudios que no se ponen de acuerdo frente al origen del drama vivido. Frente a ello, Herrera y Pertuz consideran que la dificultad para demarcar los orígenes del conflicto actual "indica a su vez las distintas temporalidades que lo han marcado y la emergencia, con base en ello, de memorias y relatos que se expresan tanto en los informes oficiales, en las producciones académicas, como en las narrativas testimoniales o en los discursos públicos" (Herrera y Pertuz, 2017:161).

Esta dificultad para establecer recortes en el tiempo para la violencia política en Colombia remite también al obstáculo para establecer acotaciones conceptuales y acontecimientos emblemáticos al respecto, traba particularmente fuerte para la escuela, que bebe de lo que se produce en otros medios. Para Sánchez la definición de violencia está asediada por la polisemia de palabras cercanas, por ello, en Colombia, no solo es un periodo histórico, también "es una forma particular de guerra caracterizada por la pluralidad de procesos y no por simples relaciones binarias (...), es pues, una guerra sin nombre" (Sánchez, 2014:43). Quizá poco abordada en la formación docente y muy recortada en los libros de texto, la violencia política ingresa a la escuela bajo la sombra de un amplio espectro de matices sin horizonte claro, sin consenso respecto a los hechos emblemáticos que la delimitan y con un manojo nefasto de protagonistas que la atraviesan (Arias, 2017). Grupos armados, hechos grandilocuentes aislados, golpes espectaculares y personajes emblemáticos hacen parte de la saga de la violencia en las memorias de la mayoría de docentes encargados de transmitirle a las nuevas generaciones las representaciones del pasado reciente, más preocupados en demostrar a sus estudiantes los fragmentos de un sino trágico que las aristas de una trama compleja que se hubiera podido evitar.

De lo anterior se pueden colegir varios asuntos, en primer lugar se ratifica que la categoría historia reciente y su enseñanza no se encuentra posicionada en el ámbito académico y escolar del país, pues, como se observa, ella remite a una argamasa de acontecimientos violentos sin orden ni estructuración común. En cambio, en otros países que padecieron dictaduras, hubo un mediano consenso social y político de desaprobación respecto a ese pasado, y el término historia reciente se refiere claramente a dicho periodo y cuentan con un acumulado para pensar el tema desde la escuela (Herrera y Pertuz, 2016). Mientras, en Colombia, inmersa aún en el conflicto armado en medio de diálogos de paz, apabullada por un fascismo societal (Santos, 1998) ${ }^{4}$, y con las formalidades de una democracia liberal, no existe aún la posibilidad de considerar pasada la página y establecer qué tiempos y actores causaron los males vividos.

En segundo lugar, para algunos docentes, aunque es loable el pretender amilanar todas las injusticias y agresiones con que llegan los estudiantes a la escuela, al no considerar la especificidad de la violencia política con todas sus ramificaciones como materia de estudio escolar, contribuyen a diluir las responsabilidades de los culpables de atrocidades, a invisibilizar actores, perder matices y apoyar la

Clín \& Asociados. La historia enseñada. Julio-Diciembre 2018 (27) ISSN 2362-3063 (digital), pp. 18-29. UNL - UNLP 
impunidad al sumarse a las versiones hegemónicas que cubren con una enorme y genérica capa semántica los infortunios de las décadas pasadas, en las que se dice que no hay inocentes en lo que pasó y que lo mejor es olvidar para rebasar rápidamente ese nefasto periodo de la historia.

A las dificultades para periodizar y tematizar el conflicto interno en la escuela se le pueden agregar otras causas educativas: el carácter marginal que el tema ocupa en el currículo oficial, como se mencionó atrás, específicamente en los documentos de política pública educativa y en los libros de texto; el ser un subtema optativo en la tradición de los planes escolares de noveno grado usualmente relegado para las últimas semanas del último periodo escolar; la falta de consenso de los académicos respecto a los recortes de la historia de violencia política; y la poca relevancia que ciertos docentes le otorgan a su tratamiento en las clases de ciencias sociales, hecho que se refleja en la improvisación, en carencia de planeación, o en la idea que preparar actividades sobre lo sucedido en las últimas décadas demanda demasiado esfuerzo y compromiso.

\section{Las fuentes para el trabajo escolar sobre violencia política}

Para la enseñanza de temas afines al pasado reciente los profesores hacen uso de variados materiales, entre ellos, los libros como soporte teórico para apoyar sus afirmaciones. Las ciencias sociales escolares, al momento de tratar problemas históricos, suelen auxiliarse en la productividad de los saberes disciplinares para transmitir este tipo de conocimiento. Sin embargo, la historia de la enseñanza de las ciencias sociales en Colombia, demuestra que buena parte de su trasegar ha estado al margen de preocupaciones meramente académicas (Álvarez, 2013; Rodríguez, 2017), fundamentalmente en favor de proyectos sociales y políticos determinados.

El tipo de material apropiado por los maestros para sus clases es adaptado de acuerdo a sus propósitos y al contexto en el que se encarna, de allí que se mantenga una tensión no resuelta respecto a si en el aula se produce un tipo de conocimiento propio (Goodson, 1991), o si allí se hace más bien la transposición de un saber elaborado por otros (Chevallard, 1991). Más allá de este dilema, es pertinente el análisis del tipo de fuentes y las principales obras que los docentes de ciencias sociales invocan como soporte para tratar en sus clases los contenidos de la violencia política en Colombia.

Frente a la enorme cantidad de material que se ha producido en los últimos años sobre el tópico de la violencia política y el conflicto social y armado en Colombia hay que resaltar que todavía no se da una apropiación masiva en los escenarios escolares. Esta fractura es preocupante, pues este vacío abre la pregunta sobre ¿de dónde se nutren las versiones escolares sobre el pasado reciente? Con poco tiempo en el calendario escolar, sin acuerdo respecto a los aspectos a tratar y con una notoria pobreza de fuentes para su manejo en el aula no hay que ir muy lejos para imaginar que los relatos escolares sobre el pasado reciente probablemente están llenos de sentido común, de información inexacta y de sesgos ideológicos propios de escenarios como la televisión privada y los libros de texto.

También hay que indicar que la copiosa producción académica sobre el tema poco es pensada para el trabajo en la educación básica y media, y que, por tanto, se requieren más propuestas serias elaboradas por equipos de pedagogos que tengan en cuenta las particularidades de la escuela, sus ritmos, tiempos y destinatarios. Por otro lado, hay que señalar en este punto la importancia sobre el uso de diferentes estrategias audiovisuales a las que acceden los profesores, especialmente videos y documentales, lo cual da cuenta de la urgencia educativa por transmitir mensajes escolares con mediaciones cercanas a las nuevas generaciones.

En lo referente al uso del libro de texto como fuente para la enseñanza de estos tópicos, es claro que este constituye una herramienta clave en el desarrollo de las clases de todas las asignaturas escolares. Algunos países lo distribuyen gratuitamente entre los estudiantes, y en otros contextos la regulación por parte del Estado pone en evidencia lo estratégico que puede ser para determinar los aprendizajes escolares. Los usos que dicen practicar los docentes de los libros de texto es múltiple y variado. Muchos profesores son conscientes de las limitaciones que exhibe este tipo de material, sobre todo en 
relación con los aspectos que estos desconocen y con la información parcializada que proyectan, de allí que lo utilicen como un apoyo o un complemento en la rutina de sus clases.

\section{Conclusiones}

Uno de los hallazgos principales de los estudios aquí expuestos consiste en ratificar que la categoría historia reciente no está posicionada en el imaginario docente para aludir a determinado pasado, como sí ocurre en otros países que tuvieron dictaduras. Por lo general, bajo el paraguas del término violencia política o pasados en conflicto se agrupa un conjunto disímil de temas que no se limita al siglo XX o al territorio nacional. En otras palabras, no existe en la escuela colombiana un perfil acotado para nombrar el reciente pasado signado por el conflicto interno. No hay aún una historia traumática que se mire a la distancia, como parte de un periodo pretérito, entre otras cosas, por la existencia de unos diálogos con la insurgencia en medio de la confrontación, y por pervivencia de sistemáticas violaciones a los derechos humanos de líderes de oposición ${ }^{5}$. El hecho que aún se dé el accionar de las bandas criminales, de grupos paramilitares y sus alianzas con las fuerzas armadas legales, así como la pervivencia de grupos insurgentes, permite pensar que en el país aún no se está en la senda de una paz "estable y duradera", como reza el eslogan del gobierno de Santos, pese a la importancia que representa la desmovilización de las FARC.

Adicionalmente, como sucede con la violencia en Colombia, que desborda los cauces de las razones ideológicas, buena parte de los docentes considera que esta no tiene límites temporales ni espaciales claros, para algunos, incluso, parece inscrita en la genética de los pobladores, y dicha representación se traslada a sus prácticas pedagógicas.

Respecto a los tiempos que los docentes dedican al tema, la mayoría dice alcanzar a tratarlo someramente en clase, y ocupan, en el mejor de los casos, dos semanas del calendario académico. Pocos docentes dedican más de un mes para trabajarlo con sus estudiantes. Los temas recurrentes reportados por los docentes sobre el pasado reciente, si bien bordean lo sucedido en el siglo XX, tocan también eventos anteriores y dramas de otros continentes. Casi ningún profesor se refirió a coyunturas actuales como los diálogos de paz con la insurgencia o la Cátedra de paz, sin embargo, los pocos que lo hicieron se refirieron a la transmisión de estos eventos en el aula de manera profusa y didácticamente innovadora. Respecto a los espacios, su enseñanza continúa muy acotada al espacio del aula sin que se aprovechen otros escenarios escolares u otros sitios relacionados con los lugares de la memoria que han empezado a institucionalizarse en las últimas décadas.

Sobre las fuentes utilizadas por los docentes, la mayoría declara hacer uso selectivo de libros de texto, y pocos se niegan a usarlos por considerarlos sesgados y reduccionistas en el tratamiento de la violencia política. Hubo importantes referencias a obras de carácter académico para apoyar la labor pedagógica, especialmente el libro Colombia: una nación a pesar de sí misma, de David Bushnell (1994), aunque fue pobre la mención de obras especializadas elaboradas por ONGs o el Estado destinadas a abordar las vicisitudes del conflicto como materia escolar. También destacaron como fuentes el uso de películas, documentales, material de Internet y, en menor medida, obras de literatura.

La enseñanza de la historia reciente en Colombia, vale decir, de la violencia política y el conflicto interno mirado desde el aula de educación formal, es un espacio en construcción que concita el interés de varios actores cuyas posibilidades de generalización en las aulas del país aún es incipiente por muchas razones, entre otras, por la permanencia del conflicto en muchas zonas, por la falta de una agenda pública que posicione con más fuerza el tema en el itinerario de las instituciones sociales, los medios y la academia, y también por la tibieza y ambigüedad con el que la política educativa lo trata.

Proscrito de los libros de texto y marginal en las apuestas curriculares de noveno grado, el pasado reciente es la trinchera pedagógica de algunos docentes comprometidos con el cambio social, que trascienden en su práctica las limitaciones de los planes prescriptivos, pero también, para otros, es la excusa de la formación axiológica en un medio social precario que pauperiza las nuevas generaciones.

Clín \& Asociados. La historia enseñada. Julio-Diciembre 2018 (27) ISSN 2362-3063 (digital), pp. 18-29. UNL - UNLP 
En suma, el estudio del conflictivo pasado colombiano le sirve a los profesores para muchas cosas, todas ellas determinadas por sus propias biografías. Queda esperar que este contenido escolar a futuro se cualifique, más allá de los gustos de los educadores, no solo por la posibilidad demostrada que brinda para formar políticamente a ciudadanos en la escuela más conscientes de su realidad, y por tanto con posibilidad de mejorarla, o porque contribuya a rearmar la propia subjetividad docente desde otros referentes, sino porque aporta a pensarnos y narrarnos como sociedad más allá de la guerra.

\section{Bibliografía}

Álvarez, A. (2013). Las ciencias saciales en Calambia. Genealagías pedagógicas. Bogutá: Instituto para la Investigación Educativa y el Desarrolla Pedagúgico.

Arias, D. \& Ruiz, A. (2013). Jóvenes, política e identidad nacional. Un estudio can jóvenes universitarios colambianas. Revista Argentina de Estudios de Juventud, 7, I-22.

Arias, D. (2005). Enseñanza y aprendizaje de las ciencias saciales. Bogatá: Cooperativa Editorial Magisterio.

Arias, D. (2015). La enseñanza de las ciencias saciales en Colambia: lugar de las disciplinas y disputa par la hegemanía de un saber. Revista de Estudias Saciales, 52: 134-146.

Arias, D. (2017). Relatos de nación y escuela. Culombia en los imaginarios de dacentes en farmación. Bugatá: Universidad Distrital Francisco Jasé de Caldas.

Bushnell, D. (1994). Calambia, una пасíńn a pesar de sí misma. De las tiempas precolambinas a nuestros días. Bugatá: Planeta.

Carretero, M. \& Borrelli, M. (2008). La enseñanza de la historia reciente: propuestas para pensar históricamente. En Саггеteru, M. \& Castorina, 」. (eds.). La canstrucción de/ canacimienta histórica. Enseñanza, narración e identidades (IDI130). Buenos Aires: Paidós.

Carretero, M. (2005). Canstruir y enseñar las ciencias saciales y la histaria. Buenos Aires: Aique.

Chevallard, Y. (1991). La transposición didáctica: de/ saber sabio al saber enseñada. Buenos Aires: Aique.

De Amézola, G. (2008). Currículo oficial y memoria. El pasada reciente en la escuela argentina. Enseñanza de las ciencias saciales, 7, 47-55.

Dussel, I. (2002). La educación y la memoria. Notas sobre la política de la transmisión. Revista Anclajes, 6, 267-293.

Escolano, A. (2000). Tiempos y espacios para la escuela. Madrid: Bibliateca Nueva.

Falaize, B. (2010). El métoda para el análisis de los temas sensibles de la histaria. En Ávila, R., Riveru, P. \& Damínguez, P. (courds.). Metadalagía de investigación en Didáctica de las Ciencias Saciales (187-205). Zaraguza: Institución Fernanda El Católico.

Funes, P. (2006). Sa/var la nación. Intelectuales, cultura y política en los años veinte latinaamericanos. Buenus Aires: Prometeo.

Gimeno, J. \&̊ Pérez, A. (1993). Camprender y transformar la enseñanza. Madrid: Morata.

Gimeno, J. (1998). Paderes inestables en educación. Madrid: Morata.

Goudson, I. (1991). La construcción sacial del currículum. Pasibilidades y ámbitas de investigación de la histaria del currículum. Revista de Educación, 295, 7-37.

Guzmán, G., Fals, Q. \& Umaña, L. (2010). La violencia en Colombia. Bugatá: Alfaguara.

Herrera, M. a Pertuz, L. (2017). Educación y palíticas de la memaria en América Latina. Par una pedagagía más allá del paradigma de/ sujeta víctima. Bogatá: Universidad Pedagúgica Nacional.

Herrera, M. \& Vélez, G. (2014). Formación política en el tiempa presente: ecolagías violentas y pedagagía de la memoria. Nómadas, 41, 149-165.

Herrera, M., Drtega, P., Cristancho, J. \& Dlaya, V. (2013). Memaria y formación: configuracianes de la subjetividad en ecologías vinlentas. Bugatá: Universidad Pedagúgica Nacional. 
Higuera, D. (2012). La escuela ante la transmisión del pasado reciente argentina: sentidas camunes, dilemas de la representación y los desafíos de/ presente. Buenos Aires: Libros libres, FLACSD.

Jelin, E. \& Lorenz, F. (comps.) (2004). Educación y Memoria. La escuela elabora e/pasado. Buenos Aires: Siglo XXI.

Jelin, E. (2003). Memorias y luchas políticas. En Degregari, ¿. (ed.). Jamás tan cerca arremetió lo lejos. Memoria y violencia politica en e/ Perú (27-48). Lima: Instituto de Estudios Peruanos.

Kriger, M. (2011). La enseñanza de la historia reciente como herramienta clave de la educación política. Persana y Saciedad, 3, 29-52.

Levín, F. (2007). El pasado reciente en la escuela, entre los dilemas de la historia y la memoria. En Schujman, G. \& Siede, I. (eds.). Ciudadanía para armar. Aportes para la formación ética y política (157-178). Buenos Aires: Aique.

Meirieu, P. (2004). En la escuela hay. Barcelona: Dctaedro.

MEN (2002). Lineamientas curriculares para el área de ciencias saciales. Serie lineamientas curriculares. Bogotá: Ministerio de Educación Nacional.

MEN (2004). Estándares básicas de ciencias saciales. Bogotá: Ministerio de Educación Nacional.

MEN (2017). Derechos Básicas de Aprendizaje de ciencias saciales. Dacumenta barradar. Bogatá: Ministerio de Educación Nacional.

Mendoza, N. (2015). Paliticas de la memoria y transmisión generacional de pasados recientes. México: Universidad Nacional Autónoma de México.

Pagés, J. \& Santisteban, A. (eds.) (2013). Una mirada al pasado y al proyecto de futuro. Investigación e innavación en didáctica de las ciencias saciales. Barcelona: UAB.

Palacios, M. \& Safford, F. (2002). Calombia: país fragmentada, saciedad dividida. Bogotá: Norma.

Rodríguez, S. (2017). Memaria y a/vida: usas públicas de/pasado en Calombia, 1930-1960. Bogatá: Universidad Nacional de Colombia/ Universidad del Rosario.

Sánchez Gómez, G. (1990). Guerra y política en la sociedad colombiana. Análisis politica, 11, 7-33.

Sánchez, G. (2014). Guerras, memuria e historia. Medellín: La Carreta.

Santos, B. (1998). Reinventar la demacracia. Reinventar e/ Estada. Lisboa: Gradiva.

\section{Notas}

${ }^{1}$ Producto de dos investigaciones: 1) "Perspectivas docentes sobre enseñanza de la historia reciente en Colombia", financiada por el Centro de Investigaciones y Desarrollo Científico de la Universidad Distrital Francisco José de Caldas (Cód. 2-7-535-16), realizada entre 2016 y 2017 por Diego Arias. 2) "Programa de Investigación y Formación Configuración de subjetividades y constitución de memorias sobre la violencia política en América Latina" financiado por la Universidad Pedagógica Nacional (Bogotá-Colombia) y su Centro de Investigaciones (CIUP) (Cód. DPG-449-17), realizada en 2017 por Martha Cecilia Herrera y Vladimir Olaya.

${ }^{2}$ En Colombia, hasta 1984 se enseñaba historia y geografía como áreas independientes en la educación básica. A partir de ese año se propendió por una integración curricular mediante el Decreto 1002/1984. Por su parte, la Ley 115/1994 define como área obligatoria y fundamental de la educación básica en un solo enunciado a Ciencias sociales, historia, geografía, constitución política y democracia. A su vez, los Lineamientos curriculares mutan hacia una orientación interdisciplinar, mientras que los Estándares se devuelven porque, aunque no se declaran disciplinares, optan por unas matrices por grado que privilegian ciertos contenidos asociados a las asignaturas tradicionales, al igual que sucede con los recientemente llamados Derechos Básicos de Aprendizaje.

${ }^{3}$ Una de las primeras comisiones oficiales de estudios en la violencia cuyo informe salió al público en 1962, indicaba que la violencia era uno de los peores males de la sociedad colombiana y que a pesar de tener antecedentes históricos antes de 1948, es esta fecha la que inaugura el periodo conocido como La Violencia. Entre los principales propósitos del estudio estaba "ser una campanada que al redoblar hiera la sensibilidad de los colombianos y los obligue a pensar dos veces antes de volver a estimular el ciclo de la destrucción inútil y de la sevicia rebosante que se inició en 1949" (Guzmán, Fals y Umaña, 2010:27). Por su parte, Sánchez Gómez, pese a considerar La Violencia como una especie de continuidad con las catorce guerras civiles del siglo XIX que no eran "negación o sustituto, sino prolongación de las relaciones políticas" (1990:16), afirma que aquella introduce como novedad una creciente confrontación entre clases dominantes y clases subalternas que coexiste con otras oposiciones de clases como la populista y la partidista, y que dicho periodo contiene tres componentes

Clín \& Asociados. La historia enseñada. Julio-Diciembre 2018 (27) ISSN 2362-3063 (digital), pp. 18-29. UNL - UNLP 
que la hacen particular y clave para explicar lo que vendrá: la violencia como terror concretado, la violencia como resistencia armada y la violencia como conmoción social. Para Palacios y Safford el término Violencia alude a el periodo que va de 1946 a 1964, "en estos años se partió en dos el siglo XX colombiano. Las variaciones de los cálculos estadísticos ofrecidos, que van de 80.000 a 400.000 muertos, revelan el cariz partidista" (Palacios y Safford, 2002:630).

${ }^{4}$ Según el autor se trata de una nueva forma de fascismo que armoniza la democracia y el capitalismo, cuyas características son: la segregación social de los excluidos; el surgimiento de un Estado paralelo que actúa de manera diferenciada para las zonas salvajes y para las civilizadas; el fascismo paraestatal que consiste en la usurpación o neutralización del control social producido por el Estado (contratos precarios, privatización); el fascismo populista o democratización de estilos de vida fuera del alcance de la mayoría de la población; el fascismo de la inseguridad que es la manipulación a los sectores más débiles hacia la incertidumbre y ansiedad respecto al presente y el futuro; y el fascismo financiero es la economía de la especulación cuyas lógicas y consecuencias escapan del control de las mayorías.

${ }^{5}$ A marzo de 2017 la Defensoría del Pueblo denuncia el asesinado de 156 líderes sociales en los últimos 14 meses, también cinco desapariciones forzadas y 33 atentados. Si bien las confrontaciones armadas disminuyeron ostensiblemente, los homicidios selectivos han aumentado. Fuente: http://www.elpais.com.co/colombia/registran-156-asesinatos-de-lideressociales-en-colombia-en-los-ultimos-14-meses.html (09-03-2017). 